\title{
In silico assessment of interaction of sea anemone toxin APETx2 and acid sensing ion channel 3
}

\author{
Taufiq Rahman*, Ewan St. John Smith \\ Department of Pharmacology, University of Cambridge, Tennis Court Road, Cambridge CB2 1PD, UK
}

\section{A R T I C L E I N F O}

\section{Article history:}

Received 20 May 2014

Available online 2 June 2014

\section{Keywords:}

ASIC3

Protein-protein docking

Homology modelling

APETx2

\begin{abstract}
A B S T R A C T
Acid sensing ion channels (ASICs) are proton-gated cation channels that are expressed throughout the nervous system and have been implicated in mediating sensory perception of noxious stimuli. Amongst the six ASIC isoforms, ASIC1a, 1b, 2a and 3 form proton-gated homomers, which differ in their activation and inactivation kinetics, expression profiles and pharmacological modulation; protons do not gate ASIC2b and ASIC4. As with many other ion channels, structure-function studies of ASICs have been greatly aided by the discovery of some toxins that act in isoform-specific ways. ASIC3 is predominantly expressed by sensory neurons of the peripheral nervous system where it acts to detect acid as a noxious stimulus and thus plays an important role in nociception. ASIC3 is the only ASIC subunit that is inhibited by the sea anemone (Anthopleura elegantissima)-derived toxin APETx2. However, the molecular mechanism by which APETx2 interacts with ASIC3 remains largely unknown. In this study, we made a homology model of ASIC3 and used extensive protein-protein docking to predict for the first time, the probable sites of APETx2 interaction on ASIC3. Additionally, using computational alanine scanning, we also suggest the 'hot-spots' that are likely to be critical for ASIC3-APETx2 interaction.
\end{abstract}

(c) 2014 Elsevier Inc. All rights reserved.

\section{Introduction}

Acid-sensing ion channels (ASICs) are voltage-insensitive, proton-gated cation channels that belong to the epithelial sodium channel (ENaC)/degenerin (DEG) family of ion channels. ASICs are expressed in both the central and peripheral nervous system and, due to their ability to respond to acidosis, they have been implicated in nociception associated with inflammation and ischemia [1].

Four genes encode six ASIC proteins (ASIC1a, 1b, 2a, 2b, 3 and 4; the genes encoding ASIC1 and ASIC2 have splice variants that produce a and b subtypes). Functional ASICs can be either homotrimers or heterotrimers, although neither ASIC2b nor ASIC4 form functional homomers [2]. Subunit composition dictates numerous channel properties including $\mathrm{pH}$ sensitivity, activation and inactivation kinetics and pharmacological modulation [2]. Unlike ASIC1 and ASIC2, which are expressed throughout the nervous system, ASIC3 expression is restricted largely to the periphery. Electrophysiological evidence proposes that ASIC3 is a key candidate for sensing the acidosis that occurs during episodes of myocardial ischaemia and there is strong electrophysiological and behavioural evidence for a role of ASIC3 in sensory mechanotransduction $[3,4]$,

\footnotetext{
* Corresponding author. Fax: +4401223 334000

E-mail address: mtur2@cam.ac.uk (T. Rahman).
}

although ASIC3 itself is not mechanosensitive $[5,6]$. In addition to protons, endogenous, modulators of ASIC3 include the membrane protein stomatin, which acts as a dimer to inhibit ASIC3 [7] and agmatine, which causes sustained activation of ASIC3 at neutral $\mathrm{pH}$ [8]. Structure function studies have been greatly aided by the discovery of the first peptide ligand of ASIC3 channels from the toxin of the sea anemone Anthopleura elegantissima [9]. This toxin, known as APETx2 is a 42 amino acid peptide that selectively blocks ASIC3 homomeric channels as well as heteromeric ASIC $2 b+3$, ASIC1b+3, and ASIC1a+3 channels with decreasing affinities [8]. Unlike toxins such as psalmotoxin 1 (PcTx1) and mambalgin 2, which modulate ASIC1 subunits [10-13], little is known about the precise binding site of APETx2 on ASIC3. In this work, we sought to predict the possible site of interaction of APETx2 with ASIC3 using extensive molecular modelling, protein-protein docking and computational alanine scanning mutagenesis.

\section{Methods}

\subsection{Homology modelling of rASIC3}

The homology model of homomeric rat ASIC3 (rASIC3, Uniprot accession: 035240) was built using Modeller 9.11 [14] from the $1.9 \AA$ crystal structure of chicken ASIC1 homotrimer (PDB id 2QTS) [15] as a template. Target and template sequences were 
aligned according to Jasti et al. [15] using ClustalW2 (http://www. ebi.ac.uk/Tools/msa/clustalw2/). Initially, 50 models of rASIC3 were generated and the best model was chosen based on the DOPE score implemented within Modeller [14]. For the chosen model, addition of missing hydrogen atoms and imposition of protonation of histidine and acidic residues were done using the program REDUCE [16]. The model was then embedded into a pre-equilibrated membrane bilayer consisting of 128 molecules of 1-palmitoyl-2-oleoyl-phosphatidylcholine (POPC). The system was then solvated in an octahedral box with TIP3P water molecules and neutralized by adding $\mathrm{NaCl}$ up to $0.15 \mathrm{M}$. Afterwards the system was energy-minimised by a round of steepest descent minimisation followed by 500 steps of simulated annealing. Finally, to relieve the rASIC3 model of any conformational strain likely to be imposed by the template used, a 500 ps of unrestrained MD simulation was carried out at $298 \mathrm{~K}$ in YASARA structure suite (version 13.8.4, Yasara Biosciences GmbH, Austria) [17] using the AMBER03 force field [18]. Particle-mesh Ewald (PME) summation [19] for the electrostatics was used for long-range Coulomb interactions with a cut-off of $7.86 \AA$. Finally, the overall quality of rASIC3 model was assessed using PROCHECK [20] and the ERRAT server [21].

\subsection{Preparation of the toxin structures}

The structures of PcTx1 [22] and APETx2 were obtained from the available NMR-derived structures (pdb ids 2KNI and 1WXN, respectively) [23]. Further refinement of the toxin structure was made in the YASARA structure suit by side chain optimisation and energy minimisation, keeping the backbone fixed. Side chain optimisation was performed using the SCWALL method [24] whilst YAMBER2 force field [25] was used for energy minimisation. After removal of conformational stress by a short steepest descent minimisation, the procedure continued by simulated annealing (time step $2 \mathrm{fs}$, atom velocities scaled down by 0.9 every 10 th step) until convergence was reached.

\subsection{Optimisation of protein-protein docking protocol}

Initially, several protein-protein docking servers were tried and these include ClusPro 2.0 (http://cluspro.bu.edu), ZDOCK (http://zdock.umassmed.edu), Hex (http://hexserver.loria.fr), GRAMM-X 1.2.0 (http://vakser.bioinformatics.ku.edu/resources/ gramm/grammx/) and FTDock accessed through PyDockWeb (http://life.bsc.es/servlet/pydock/home/). For judging the performance the algorithms and scoring functions implemented in these servers, a cognate blind-docking approach was used by redocking of PcTx1 to chicken ASIC1a trimer and comparing the output docked pose of the PcTx1 with the original pose present in the crystal structure (pdb: 4FZ0) [11]. The ASIC1a low pH structure (pdb: 4FZO) was energy-minimised with the bound PcTx1 removed. The algorithm (s) offering the least positional difference (indicated by the root means squared deviation, r.m.s.d value) between the docked and original PcTx1 structure was considered the most reliable and thus was chosen for docking APETx2 onto rASIC3 model. The top ranked docked complex was submitted to RosettaDock [26] for further refinement. The lowest-energy complex was chosen from the out-puts from RosettaDock as the final toxin-bound channel structure.

\subsection{Other methods}

Robetta server (http://robetta.bakerlab.org/) was used for the computational alanine-scanning mutagenesis [27]. The electrostatic surface potential maps were generated using the Adaptive
Poisson-Boltzmann Solver (APBS) [28]. All graphics were prepared using PyMol.

\section{Results}

\subsection{Quality of the homology model}

As most of the experimental studies of APETx2-mediated inhibition of ASIC3 channels have been conducted on rASIC3, we considered this isoform for the present study. The rASIC3 model (Fig. 1A) was broadly similar to the template cASIC1a (PDB 2QTS) used. Individual chains superimpose well (r.m.s.d $<1.5 \AA$ ) (Fig. 1B) on each other, which is unsurprising, given the considerable ( $64 \%)$ homology in their amino acid sequences. Trajectory analysis following brief ( $500 \mathrm{ps}$ ) MD simulation of the membrane-embedded rASIC3 model indicates that the structure largely stabilized after 200 ps as evidenced by stability of the r.m.s.d values of the backbone atoms with respect to those of the starting structure (Fig. 1C).

The rASIC3 model (Fig. 1B) used for the present work seemed to have good stereo-chemical features when assessed by the Ramachandran plot analysis. The result of this analysis (Fig. S1) indicated $93.2 \%$ of rASIC3 residues were in the most favoured regions, 5.4\% was in an additionally allowed region, $1.4 \%$ was in generously allowed region with none within the disallowed region. The model was also assessed by ERRAT score, which gives an overall quality factor for non-bonded atomic interactions, and a score of greater than 50 is acceptable [21]. The template cASIC1 and our final refined model of rASIC3 yielded ERRAT scores of 87.40 and 72.05 , respectively, and the values were well within the range of high quality.

\subsection{Protein-protein docking}

To find the best docking protocol for our protein partners, we first considered several web servers such as ZDOCK [29], ClusPro 2.0 [30], GRAMM-X 1.2.0 [31], Hex [32], PatchDock [33] and FTdock accessed through PyDockWeb (http://life.bsc.es/servlet/pydock/ home/). Of these docking severs, ClusPro, GRAMM-X, ZDOCK and FTdock exploit fast-Fourier transform methods for grid matching whereas PatchDock relies on shape complementarity and symmetry restrictions; and Hex is based on spherical harmonic representations [34]. To find the best docking algorithm and scoring function, we tried cognate, blind docking of PcTx1 to cASIC1, for which known crystal structures exist, and we only considered the top 10 output poses from each to the above mentioned web servers. Among the latter, ZDOCK, ClusPro, FTdock and GRAMM$\mathrm{X}$ produced top ranked poses of PcTx1 that broadly lay within similar region (r.m.s.d $<4 \AA$ ) found in the original crystal structure (PDB id 4FZ0). However, in terms of the absolute proximity of the docked pose with the original pose of PcTx1, ZDOCK proved to be the best (r.m.s.d $<3 \AA$ ) (Fig. 2A) and we used ZDOCK for the initial blind docking APETx2 on rASIC3 model. Since ZDOCK essentially considers the interacting protein partners as rigid and thus excludes the possibility of any structural rearrangement occurring [29], we submitted the top ranked complex of PCTx1 and cASIC1 to the Rosetta protein-protein docking protocol [26] for further refinement. The RosettaDock server identifies low-energy conformations of a protein-protein complex near a given starting conformation through optimisation of rigid-body orientation and side-chain conformations [35]. Post-docking refinement through Rosetta docking resulted in significant improvement in PcTx1 pose as the distance between the docked structure to the original crystal structure (calculated as r.m.s.d) significantly reduced from $2.6 \AA$ to $1.81 \AA$, thus reproducing almost an identical pose of PcTx1 (Fig. 2B). Thus, our ultimate docking protocol involved a fast, initial 

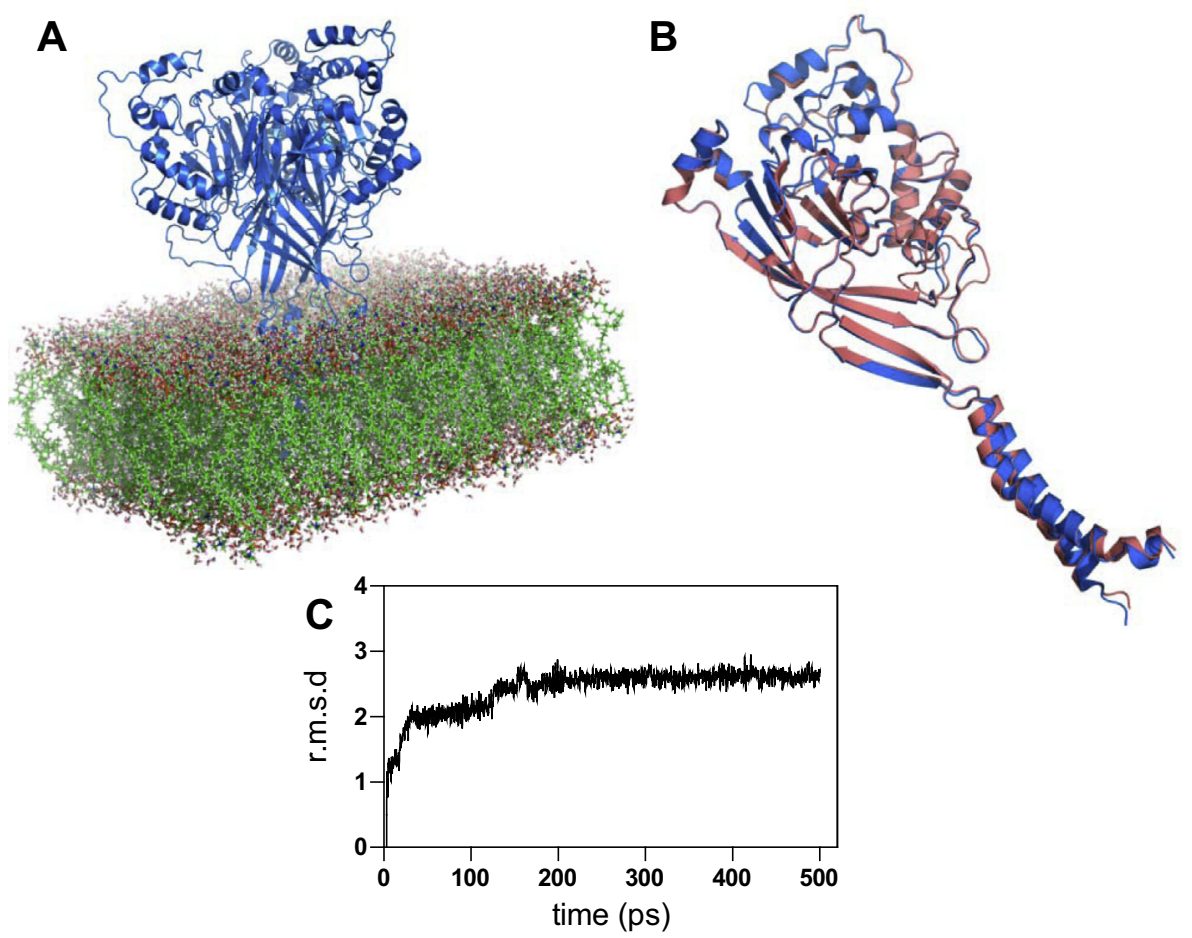

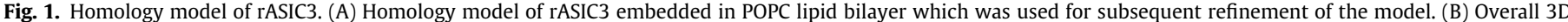

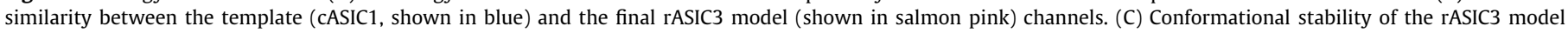

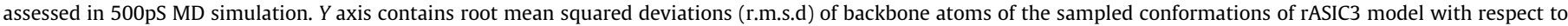
those of the initial conformation. (For interpretation of the references to colour in this figure legend, the reader is referred to the web version of this article.)
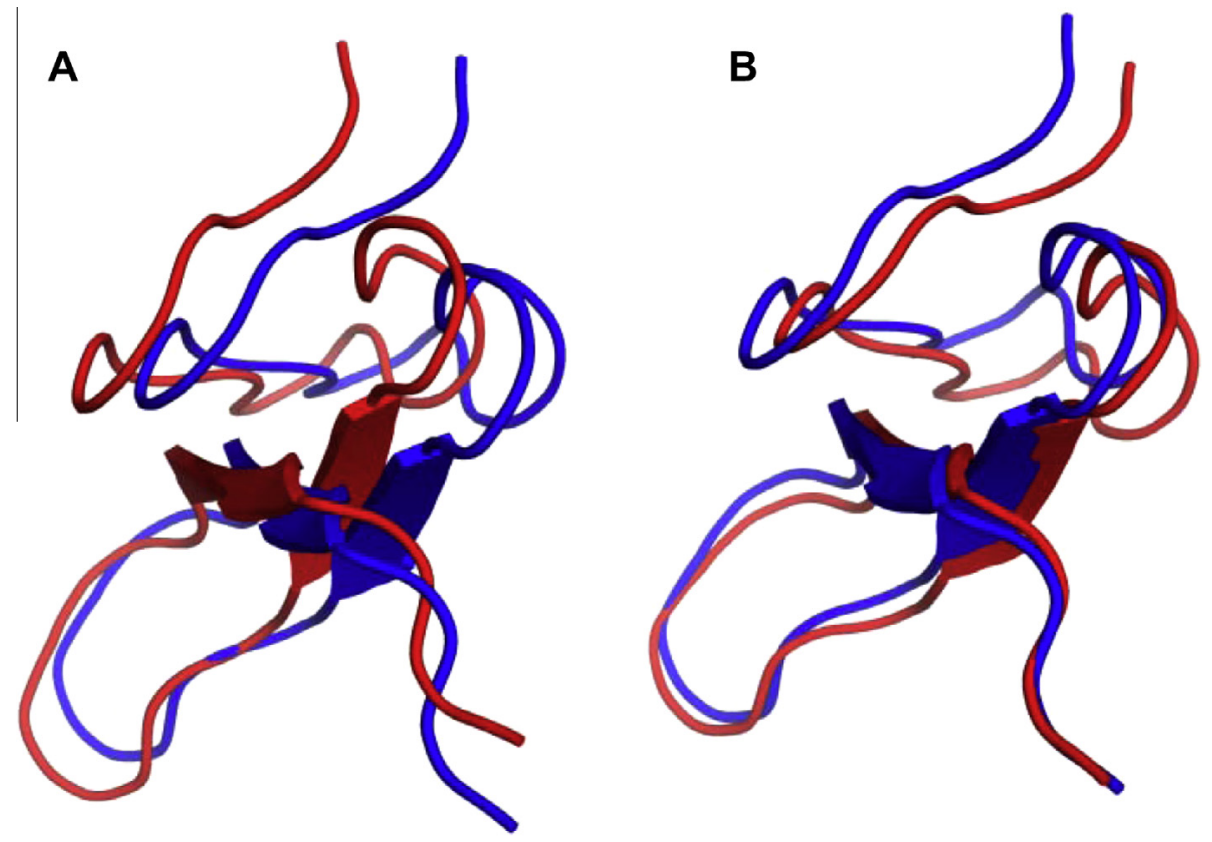

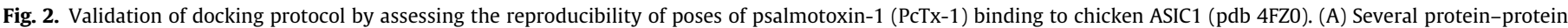

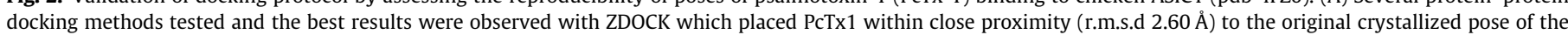

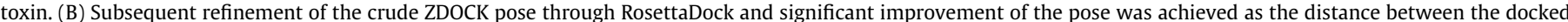

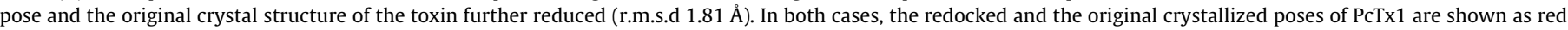
and blue, respectively. (For interpretation of the references to colour in this figure legend, the reader is referred to the web version of this article.)

rigid-body docking with ZDOCK followed by further refinement through RosettaDock.

In the top 10 ZDOCK poses, two major potential interacting sites for APETx2 were identified on the rASIC3 homotrimeric model (Fig. 3). Site A representing the highest ranked pose was within the upper 'thumb' area [36] of the extracellular domain of the channel, whilst Site B representing the second best ranked pose was between the 'wrist' and 'palm' regions, located above the trasmembrane helices (Fig. 3A and B). Clustering of poses broadly on these two sites was observed also with ClusPro, GRAMM-X and 

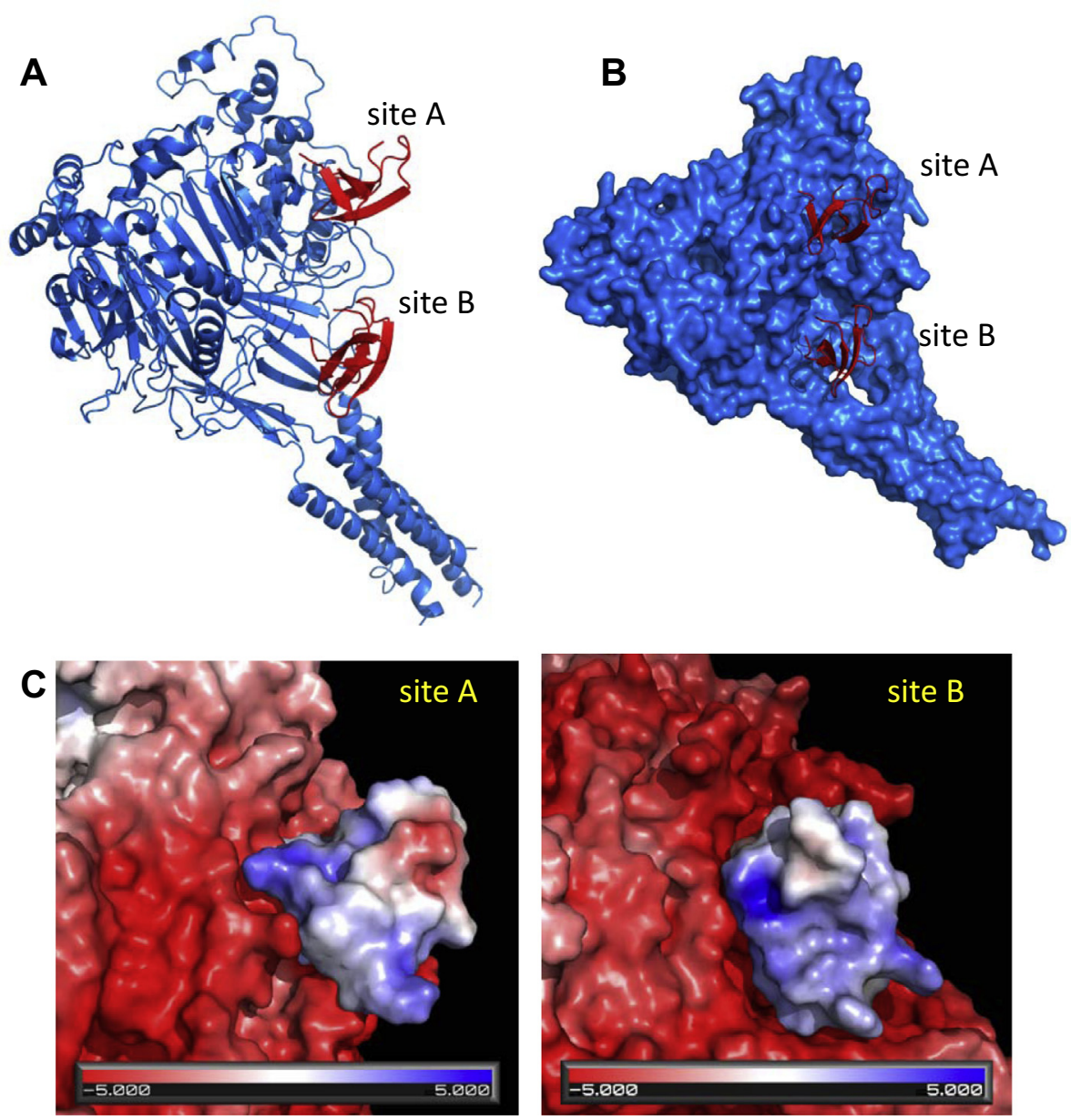

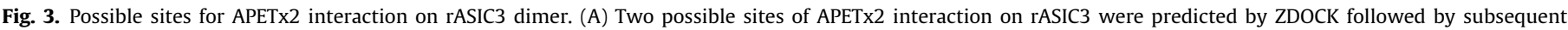

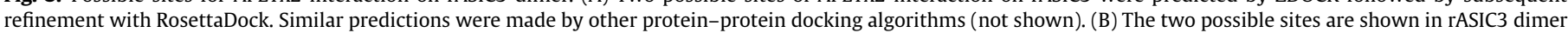

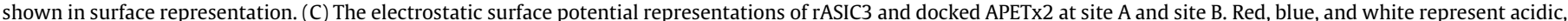

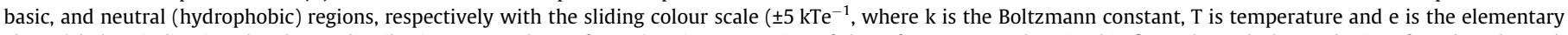

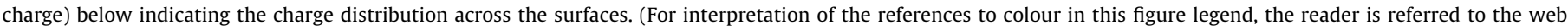
version of this article.)

FTdock (data not shown). However the relative ranking of poses at the two sites obtained through these servers varied. For example, in FTdock and GRAMM-X, Site B was ranked top, whilst Site A was second (not shown), whereas the reverse ranking was observed with ZDOCK and ClusPro.

Molecular electrostatics surface potential calculation using the APBS Solver [28] shows that both Site A and Site B on rASIC3 are largely electronegative, indicating the predominance of acidic residues within these regions (Fig. 3C). Similar analysis with APETx2 shows that the interface used by the toxin for docking on to rASIC3 at these sites is largely electropositive and electroneutral (Fig. 3C).

Next, we sought to map the key residues for the interaction between rASIC3 and APETx2 for both these sites. For this, we first identified the residues that were within 5 A distance of the docked APETx2 pose at each site and then looked for the hot spots through using the computational alanine scanning mutagenesis implemented in the Robetta server [35]. Table 1 shows the proximal residues of the interacting partners as well as the key residues that are likely to form the hot spots for the interaction (i.e. for which $\Delta \Delta G$ was greater than $1 \mathrm{kcal} / \mathrm{mol}$ ). For interaction at Site A, only F293 of rASIC3 appears to be critical whilst for site B, two potential hot spot residues - S82 and E426 are suggested. For interaction at both sites, APETx2 seems to employ almost same group of residues, which are largely hydrophobic.

\section{Discussion}

In response to reduction of $\mathrm{pH}$ from physiological $\mathrm{pH}$, the current generated by ASIC3 is typically biphasic comprising of a transient peak component followed by a slowly developing, sustained component $[7,37]$. APETx2 rapidly and reversibly inhibits the transient peak current and the sustained component evoked at $\mathrm{pH} 7.0$ [38], but it does not affect the sustained component at $\mathrm{pH} 5.0$ [9]. In several recent studies, APETx2 has also been shown to inhibit the current mediated by certain voltage-gated sodium channels with variable potencies $[39,40]$. In the present study we focused only on APETx2 modulation of ASIC3 and found two potential candidate sites on rASIC3 via which APETx2 could exert its effect. It is noteworthy that Site A is contained within the upper palm region of each subunit in homomeric rASIC3 (and a human ASIC3 model as well, data not shown). This is intriguing because it implies that the presence of a single ASIC3 subunit could confer some sensitivity towards inhibition by APETx2. Indeed, in addition to APETx2 inhibiting both rat and human homomeric ASIC3 $\left(\mathrm{IC}_{50} \mathrm{~S}\right.$ of $\sim 37-63 \mathrm{nM}$ and $\sim 175 \mathrm{nM}$ respectively), several (but not all) ASIC3-containing heterotrimeric ASICs are inhibited with lower potency $[9,23,41,42]$. Among the residues of rASIC3 that are within the 5 A distance to the docked APETx2 at Site A, only residue (F293) residue came out as a hot-spot. 
Table 1

Amino acids within the interface of rASIC3 and bound APETx2.

\begin{tabular}{|c|c|c|c|}
\hline \multicolumn{2}{|c|}{ Site A } & \multicolumn{2}{|c|}{ Site B } \\
\hline rASIC3 & APETx2 & rASIC3 & APETx2 \\
\hline T180 & $\mathrm{T} 2$ & G69 & G1 \\
\hline V181 & A3 & E70 & $\mathrm{T} 2$ \\
\hline $\mathrm{I} 182$ & F15 (1.48) & F71 & \\
\hline F183 & Y16 (2.61) & $\mathrm{H} 72$ & A3 \\
\hline A194 & P18 & W280 & W14 (2.65) \\
\hline D248 & F33 (3.69) & G281 & Y16 (2.42) \\
\hline E249 & L34 (1.31) & S82 (2.33) & \\
\hline $\mathrm{P} 250$ & & Q84 & R17(2.43) \\
\hline D292 & & $\mathrm{S} 274$ & D23 \\
\hline F293 (1.74) & & L276 & \\
\hline P295 & & A286 & R24 \\
\hline H336 & & E296 & F33 (1.07) \\
\hline M297 & & P297 & L34 (1.65) \\
\hline P338 & & E426 (1.22) & \\
\hline G339 & & $\mathrm{K} 428$ & \\
\hline N340 & & $\begin{array}{l}\text { A429 } \\
\text { A430 }\end{array}$ & \\
\hline
\end{tabular}

*The hotspot residues are shown in bold with corresponding $\Delta \Delta G>1 \mathrm{kcal} / \mathrm{mol}$ given in parenthesis. The residues of rASIC3 and APETx2 were within $5 \AA$ distance from each other. The residues coloured in red are from different subunit.

By contrast, Site B seems to lie within the 'wrist' and 'palm' region [13], which is at an interface between adjacent subunits. But only one subunit appears to contain the two predicted hot spots - S82 and E426 for this site (Table 1).

Previously, Chagot and colleagues [23] noted that two clusters of residues located on opposite faces of APETx2 were different from APETx1: Cluster 1 (A3, S5, N8, K10, T39 and A41) and Cluster 2 (Y16, R17, P18, R31 and T36). APETx1 is known to inhibit hERG channels, with no effect on ASIC3 and thus residues constituting Cluster 1 and Cluster 2 of APETx2 were proposed to be involved in the interaction and inhibition of ASIC3. Indeed, Anangi et al. [41] demonstrated that the single point mutation R17, which is part of Cluster 2, resulted in much reduced inhibition of mouse ASIC3 current. Furthermore, Jensen and colleagues [42] found that inhibition of ASIC3 by APETx2 was substantially reduced when the $\mathrm{N}$ - and $\mathrm{C}$-termini were joined through a peptide linker and also for some $\mathrm{N}$ - or C-terminally truncated mutants; truncation mutants at the $\mathrm{N}$ terminus showed the highest ( $\sim 300$-fold) reduction in ASIC3 inhibition. It was thus suggested that residues in the APETx2 N-terminus and some from Cluster 2 (Y16, R17, P18, R31 and/or T36) are essential components of the pharmacophore that mediates APETx2 interaction with ASIC3. It is noteworthy that many of the residues of docked APETx2 within 5 Å of Site A and Site B of rASIC3 model (Table 1 ) in our study overlap with those suggested by Chagot and colleagues [23] and more recently by Jensen et al. [42]. Moreover, R17, which has been reported to be critical for association of
APETx2 and mouse ASIC3, came out as a hot spot only for the Site B pose of APETx2.

Based entirely on docking and clustering of poses, both Site A and Site B remain plausible sites for APETx2 interaction with ASIC3 and Site A was ranked top when ZDOCK was used. However, several observations from published work seem to favour Site B as the preferred one. For example, Site $A$ is located near the upper 'palm' region that in ASIC1 remains effectively unchanged at variable $\mathrm{pH}$ and in toxin bound structures [13]. Furthermore, Site $\mathrm{A}$ is also located more towards the channel periphery, and quite further from the central vestibule and the ion-conduction pathway. Whether interaction at this site, perhaps allosterically, could cause the robust block of ASIC3 current by APETx2 demands experiments. Site B, on the other hand, lie just above the 'wrist' region that has been reported to be flexible and altered by two toxins (PcTx1 and Mit-Tx) that modulate the pore opening of ASIC1 $[13,43]$. Among other hot spots, APETx2 appears to use R17 to interact with Site B only; previously this residue was found to be a critical determinant of APETx2 inhibition of ASIC3 [41]. Finally, it is also intriguing to note that certain hot spots within Site B, namely S82 and E426 are proximal to residues implicated in sensing non-proton ligands of ASIC3 [44]. All these factors would indicate that of the two sites, interaction at Site B is more likely to affect channel gating and ion permeation properties. The demonstration of whether Site A or Site B is more important for APETx2 inhibition of ASIC3 must be determined through electrophysiological analysis.

\section{Conclusions}

In conclusion, we have presented a model of rASIC3 and proposed for the first time, two possible sites for APETx2 interaction and also speculated the relative plausibility of these sites. Additionally, through computational alanine scanning mutagenesis, we identified some residues that may serve as 'hot-spots' for such association at these sites. Our predictive findings should guide future mutagenesis and functional studies for mapping the actual APETx2 interaction site on ASIC3.

\section{Acknowledgment}

T.R. is a recipient of a fellowship and additional support funding (RG120370) from the Royal Society.

\section{Appendix A. Supplementary data}

Supplementary data associated with this article can be found, in the online version, at http://dx.doi.org/10.1016/j.bbrc.2014.05.130.

\section{References}

[1] J.A. Wemmie, R.J. Taugher, C.J. Kreple, Acid-sensing ion channels in pain and disease, Nat. Rev. Neurosci. 14 (2013) 461-471.

[2] T.W. Sherwood, E.N. Frey, C.C. Askwith, Structure and activity of the acid-sensing ion channels, Am. J. Physiol. Cell Physiol. 303 (2012) C699C710.

[3] R.A. Moshourab, C. Wetzel, C. Martinez-Salgado, G.R. Lewin, Stomatin-domain protein interactions with acid-sensing ion channels modulate nociceptor mechanosensitivity, J. Physiol. 591 (2013) 5555-5574.

[4] M.P. Price, S.L. Mcllwrath, J. Xie, C. Cheng, J. Qiao, D.E. Tarr, K.A. Sluka, T.J. Brennan, G.R. Lewin, M.J. Welsh, The DRASIC cation channel contributes to the detection of cutaneous touch and acid stimuli in mice, Neuron 32 (2001) 1071-1083.

[5] L.J. Drew, D.K. Rohrer, M.P. Price, K.E. Blaver, D.A. Cockayne, P. Cesare, J.N. Wood, Acid-sensing ion channels ASIC2 and ASIC3 do not contribute to mechanically activated currents in mammalian sensory neurones, J. Physiol. 556 (2004) 691-710.

[6] S.G. Lechner, H. Frenzel, R. Wang, G.R. Lewin, Developmental waves of mechanosensitivity acquisition in sensory neuron subtypes during embryonic development, EMBO J. 28 (2009) 1479-1491. 
[7] J. Brand, E.S. Smith, D. Schwefel, L. Lapatsina, K. Poole, D. Omerbasic, A. Kozlenkov, J. Behlke, G.R. Lewin, O. Daumke, A stomatin dimer modulates the activity of acid-sensing ion channels, EMBO J. 31 (2012) 3635-3646.

[8] W.G. Li, Y. Yu, Z.D. Zhang, H. Cao, T.L. Xu, ASIC3 channels integrate agmatine and multiple inflammatory signals through the nonproton ligand sensing domain, Mol. Pain 6 (2010) 88.

[9] S. Diochot, A. Baron, L.D. Rash, E. Deval, P. Escoubas, S. Scarzello, M. Salinas, M. Lazdunski, A new sea anemone peptide, APETx2, inhibits ASIC3, a major acidsensitive channel in sensory neurons, EMBO J. 23 (2004) 1516-1525.

[10] R.J. Dawson, J. Benz, P. Stohler, T. Tetaz, C. Joseph, S. Huber, G. Schmid, D. Hugin, P. Pflimlin, G. Trube, M.G. Rudolph, M. Hennig, A. Ruf, Structure of the acid-sensing ion channel 1 in complex with the gating modifier Psalmotoxin 1, Nat. Commun. 3 (2012) 936.

[11] I. Baconguis, E. Gouaux, Structural plasticity and dynamic selectivity of acid sensing ion channel-spider toxin complexes, Nature 489 (2012) 400-405.

[12] M. Salinas, T. Besson, Q. Delettre, S. Diochot, S. Boulakirba, D. Douguet, E. Lingueglia, Binding site and inhibitory mechanism of the mambalgin-2 painrelieving peptide on acid-sensing ion channel 1a, J. Biol. Chem. 289 (19)(2014) $13363-13373$.

[13] I. Baconguis, C.J. Bohlen, A. Goehring, D. Julius, E. Gouaux, X-ray structure of acid-sensing ion channel 1 -snake toxin complex reveals open state of a $\mathrm{Na}(+)-$ selective channel, Cell 156 (2014) 717-729.

[14] N. Eswar, D. Eramian, B. Webb, M.Y. Shen, A. Sali, Protein structure modeling with MODELLER, Methods Mol. Biol. 426 (2008) 145-159.

[15] J. Jasti, H. Furukawa, E.B. Gonzales, E. Gouaux, Structure of acid-sensing ion channel 1 at 1.9 A resolution and low pH, Nature 449 (2007) 316-323.

[16] J.M. Word, S.C. Lovell, J.S. Richardson, D.C. Richardson, Asparagine and glutamine: using hydrogen atom contacts in the choice of side-chain amide orientation, J. Mol. Biol. 285 (1999) 1735-1747.

[17] E. Krieger, K. Joo, J. Lee, J. Lee, S. Raman, J. Thompson, M. Tyka, D. Baker, K. Karplus, Improving physical realism, stereochemistry, and side-chain accuracy in homology modeling: four approaches that performed well in CASP8, Proteins 77 (Suppl. 9) (2009) 114-122.

[18] Y. Duan, C. Wu, S. Chowdhury, M.C. Lee, G. Xiong, W. Zhang, R. Yang, P. Cieplak, R. Luo, T. Lee, J. Caldwell, J. Wang, P. Kollman, A point-charge force field for molecular mechanics simulations of proteins based on condensed-phase quantum mechanical calculations, J. Comput. Chem. 24 (2003) 1999-2012.

[19] U. Essmann, L. Perera, M. Berkowitz, T. Darden, H. Lee, L.G. Pedersen, A smooth particle mesh Ewald method, J. Chem. Phys. 103 (1995) 8577-8593.

[20] R.A. Laskowski, E.G. Hutchinson, A.D. Michie, A.C. Wallace, M.L. Jones, J.M. Thornton, PDBsum: a web-based database of summaries and analyses of all PDB structures, Trends Biochem. Sci. 22 (1997) 488-490.

[21] C. Colovos, T.O. Yeates, Verification of protein structures: patterns of nonbonded atomic interactions, Protein Sci. 2 (1993) 1511-1519.

[22] N.J. Saez, M. Mobli, M. Bieri, I.R. Chassagnon, A.K. Malde, R. Gamsjaeger, A.E. Mark, P.R. Gooley, L.D. Rash, G.F. King, A dynamic pharmacophore drives the interaction between Psalmotoxin-1 and the putative drug target acid-sensing ion channel 1a, Mol. Pharmacol. 80 (2011) 796-808.

[23] B. Chagot, P. Escoubas, S. Diochot, C. Bernard, M. Lazdunski, H. Darbon, Solution structure of APETx2, a specific peptide inhibitor of ASIC3 protongated channels, Protein Sci. 14 (2005) 2003-2010.

[24] A.A. Canutescu, A.A. Shelenkov, R.L. Dunbrack Jr., A graph-theory algorithm for rapid protein side-chain prediction, Protein Sci. 12 (2003) 2001-2014.
[25] E. Krieger, T. Darden, S.B. Nabuurs, A. Finkelstein, G. Vriend, Making optimal use of empirical energy functions: force-field parameterization in crystal space, Proteins 57 (2004) 678-683.

[26] J.J. Gray, S. Moughon, C. Wang, O. Schueler-Furman, B. Kuhlman, C.A. Rohl, D. Baker, Protein-protein docking with simultaneous optimization of rigid-body displacement and side-chain conformations, J. Mol. Biol. 331 (2003) 281-299.

[27] T. Kortemme, D.E. Kim, D. Baker, Computational alanine scanning of proteinprotein interfaces, Sci. STKE 2004 (2004) pl2.

[28] N.A. Baker, D. Sept, S. Joseph, M.J. Holst, J.A. McCammon, Electrostatics of nanosystems: application to microtubules and the ribosome, Proc. Natl. Acad. Sci. U.S.A. 98 (2001) 10037-10041.

[29] B.G. Pierce, Y. Hourai, Z. Weng, Accelerating protein docking in ZDOCK using an advanced 3D convolution library, PLoS One 6 (2011) e24657.

[30] S.R. Comeau, D.W. Gatchell, S. Vajda, C.J. Camacho, ClusPro: an automated docking and discrimination method for the prediction of protein complexes, Bioinformatics 20 (2004) 45-50.

[31] A. Tovchigrechko, I.A. Vakser, GRAMM-X public web server for protein-protein docking, Nucleic Acids Res. 34 (2006) W310-W314.

[32] D.W. Ritchie, G.J. Kemp, Protein docking using spherical polar Fourier correlations, Proteins 39 (2000) 178-194.

[33] D. Schneidman-Duhovny, Y. Inbar, R. Nussinov, H.J. Wolfson, PatchDock and SymmDock: servers for rigid and symmetric docking, Nucleic Acids Res. 33 (2005) W363-W367.

[34] S. Grosdidier, J. Fernandez-Recio, Protein-protein docking and hot-spot prediction for drug discovery, Curr. Pharm. Des. 18 (2012) 4607-4618.

[35] S. Lyskov, J.J. Gray, The RosettaDock server for local protein-protein docking, Nucleic Acids Res. 36 (2008) W233-W238.

[36] H. Ishikita, Proton-binding sites of acid-sensing ion channel 1, PLoS One 6 (2011) e16920.

[37] M. Salinas, M. Lazdunski, E. Lingueglia, Structural elements for the generation of sustained currents by the acid pain sensor ASIC3, J. Biol. Chem. 284 (2009) 31851-31859.

[38] E. Deval, J. Noel, X. Gasull, A. Delaunay, A. Alloui, V. Friend, A. Eschalier, M. Lazdunski, E. Lingueglia, Acid-sensing ion channels in postoperative pain, J. Neurosci. 31 (2011) 6059-6066.

[39] M.G. Blanchard, L.D. Rash, S. Kellenberger, Inhibition of voltage-gated $\mathrm{Na}(+)$ currents in sensory neurones by the sea anemone toxin APETx2, Br. J. Pharmacol. 165 (2012) 2167-2177.

[40] S. Peigneur, L. Beress, C. Moller, F. Mari, W.G. Forssmann, J. Tytgat, A natural point mutation changes both target selectivity and mechanism of action of sea anemone toxins, FASEB J. 26 (2012) 5141-5151.

[41] R. Anangi, C.C. Chen, Y.W. Lin, Y.R. Cheng, C.H. Cheng, Y.C. Chen, Y.P. Chu, W.J. Chuang, Expression in Pichia pastoris and characterization of APETx2, a specific inhibitor of acid sensing ion channel 3, Toxicon 56 (2010) 1388-1397.

[42] J.E. Jensen, T. Durek, P.F. Alewood, D.J. Adams, G.F. King, L.D. Rash, Chemical synthesis and folding of APETx2, a potent and selective inhibitor of acid sensing ion channel 3, Toxicon 54 (2009) 56-61.

[43] C.J. Bohlen, A.T. Chesler, R. Sharif-Naeini, K.F. Medzihradszky, S. Zhou, D. King, E.E. Sanchez, A.L. Burlingame, A.I. Basbaum, D. Julius, A heteromeric Texas coral snake toxin targets acid-sensing ion channels to produce pain, Nature 479 (2011) 410-414.

[44] Y. Yu, Z. Chen, W.G. Li, H. Cao, E.G. Feng, F. Yu, H. Liu, H. Jiang, T.L. Xu, A nonproton ligand sensor in the acid-sensing ion channel, Neuron 68 (2010) 61-72. 